\title{
Development of Classification Indices for Diseases using Photo Plethysmography
}

\author{
S. More and P. Choudhari \\ Department of Electronics and Telecommunication, Fr. C.Rodrigues Institute of Technology, Navi Mumbai, India \\ \{Shamali973@gmail.com,pranalic75@gmail.com\}
}

\begin{abstract}
Photo plethysmography (PPG) is used to estimate the blood flow using light. Researchers from different domains of science have been working with PPG because of its advantages as non-invasive, inexpensive, and convenient diagnostic tool. Traditionally, it has been used to measure the oxygen saturation, blood pressure and for assessing autonomic functions. Recent studies emphasize the potential information embedded in the PPG waveform signal and require attention for its possible applications beyond pulse oximetry and heart-rate calculation. The current systems based on photo plethysmography signal are used mainly for heart rate and SPO2 measurement. There is always a need for non-ohmic method, which can give idea about the blood circulation quickly and easily. The proposed system would be based on the principle of photo plethysmography, which is non-invasive in nature, thus making the entire diagnosis process simpler and less traumatic to the patients during emergencies or otherwise. It can also serve as a bedside monitoring device to monitor the various vital parameters by obtaining photo plethysmogram waveform for subjects with different ailments to desciminate the diseases.
\end{abstract}

Keywords: Photoplethysmogram,acceleratedplethysmogram,diagnosis.

\section{Introduction}

Distinct diseases can be diagnosed by invasive method and non-invasive method. Important invasive methods are angioplasty, endoscopy, coronary catheterization etc. These are time-consuming, expensive and impractical for most emergency department environments. The noninvasive methods are reliable, easy to use and having good compatibility. There are several non invasive plethysmogram techniques for recording blood volume changes in any part of the body such as volume displacement, strain gauge, impedance and photo plethysmography [1].

For quick assessment of blood flow in the limbs, air displacement plethysmograph, commercially available as pulse volume recorder is commonly employed. Volume displacement plethysmography and stain gauge plethysmography have a common limitation in accurate estimation of blood flow. In both these methods 3 to 4 levels of conversions are required to finally get the electrical signals, which are recordable while assuming that all the conversions are linear throughout the process [1].Secondly, the output of pulse volume recorder and strain gauge plethysmography is non-specific to blood.

The most widely applied 24-hour monitoring technique is Electrocardiography (ECG). But in some scenarios ECG does not provide evaluation of the functioning of human heart. Simultaneous recording of ECG and of a signal that reflects central hemodynamic activity might solve this problem. Impendence cardiography (ICG)the method calls for placement of surface electrodes on the body surface of the subject, free environment from electrical noise and stringent requirement on the specifications of the instrument from the consideration of patient safety [1].

There is always a need for non ohmic method, which can give idea about the blood circulation quickly and easily. Considering the drawback of impedance plethysmography like cumbersome procedure, ohmic contact with the patient etc., Photo plethysmography (PPG) is developed for the study of blood circulation, which is described in this paper. Photo plethysmography (PPG), a simple method that allows for continuous, non-invasive determination and monitoring of heart rate, pulse transit time etc., in the intensive care units as well as for the assessment of peripheral blood flow and venous filling time in vascular laboratories [1].

B. Iyer, S. Nalbalwar and R. Pawade (Eds.)

ICCASP/ICMMD-2016. Advances in Intelligent Systems Research.

Vol. 137, Pp. 474-483.

(C) 2017- The authors. Published by Atlantis Press

This is an open access article under the CC BY-NC license (http://creativecommons.org/licens)es/by-nc/4. 


\section{Photo Plethysmography}

Photo plethysmography (PPG) also referred to as photoelectric plethysmography (PTG), is a plethysmography technique of using sensors to detect the properties of the blood flow mainly in fingertip. PPG is a simple and low-cost optical technique that can be used to detect blood volume changes in the micro vascular bed of tissue. It is used non-invasively to make measurements at the skin surface [2] [3].

The PPG technology has been used in a wide range of commercially available medical devices for measuring oxygen saturation, blood pressure and cardiac output, assessing autonomic function and also detecting peripheral vascular disease [3]. Advantage of the plethysmogram compared to the ECG is that the recording device for a plethysmogram is lighter, less bulky and easier to attach than an ECG device; hence its ambulatory use to monitor cardiac function[3].

The basic form of PPG technology requires only a few optoelectronic components: a light source to illuminate the tissue (e.g. skin), and a photo detector to measure the small variations in light intensity associated with changes in perfusion in the catchment volume [4]. Despite its simplicity the origins of the different components of the PPG signal are still not fully understood. It is generally accepted that they can provide valuable information about the cardiovascular system.

\section{Methodology}

A diagnostic structure consists of three stages: Pre-processing, features extraction, diagnosis/classification.Preprocessing stage to emphasize the desired waves;Feature extraction stage to detect the desired waves; Calculate an index or a measure using the extracted features for classification and diagnosis [4].

\subsection{System Block Diagram}

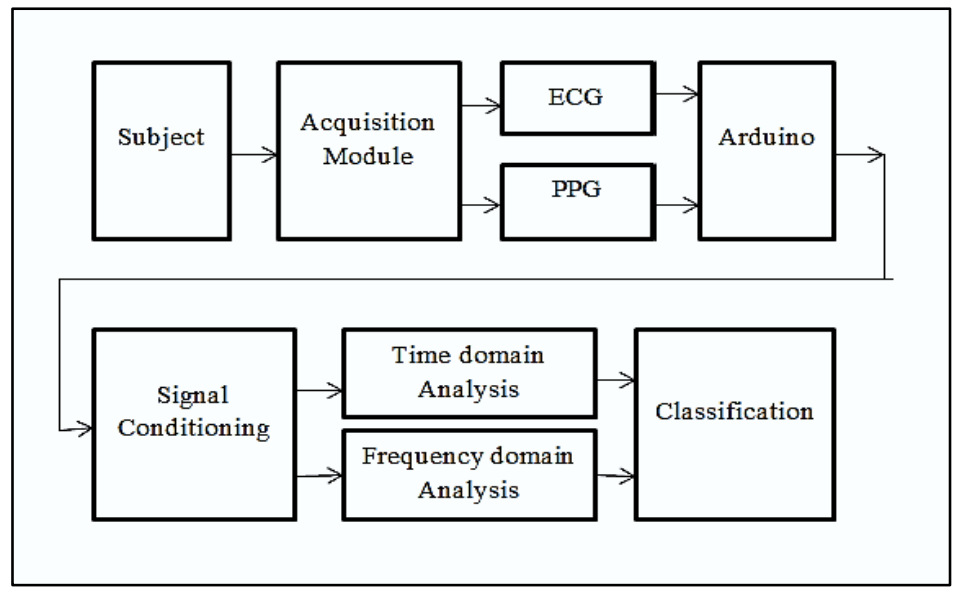

Fig. 1. System Block Diagram

Fig.1.shows the system block diagram for extraction and analysis of ECG and PPG Signals .PPG and ECG signals are collected from acquisition module are interfaced through Arduino UNO board. The signals are visualised and recorded in LabVIEW VI and then further processed and conditioned in MATLAB program. The processed signal undergoes parameter extraction and are analysed in time as well as frequency domain. After analysis, classification of diseases carried out using multi variable regression. The subjects with or without ailments are discriminated on the basis of three indices. This classification is a preliminary diagnosis which can be used to determine which tests should be performed in earlier level.

\subsection{Preprocessing}

ECG and two PPG measurements are collected simultaneously from the index finger and palm position. The raw signals were acquired from 33 subjects (with and without ailments) in normal sitting position in a chair or sleep 
comfortably on a bed in hospital conditions. The signals received from the subject were then displayed, preprocessed, analyzed and classified using MATLAB program.

The recorded signal is often contaminated by noise and artifacts that can be within the frequency band of interest and manifest with similar characteristic as the PPG signal itself. Preprocessing includes normalization, artifact removal and denoising. The major sources of artifacts in case of a fingertip signals are artifacts and movement of the patient during acquisition [4].

The sample is first processed by using normalization process which helped standardization of all features to the same level. PPG signal baselines can be shifted from zero line due to unexpected conditions like patient movements therefore it can be adjusted at zero line by subtracting the median of the PPG signal [5]. The contaminants were filtered using wavelet-transform (WT) based search algorithm which use the energy of the signal in different scales to isolate baseline wander from the PPG signal.

The algorithm explained is based on the assumption that PPG signal and baseline drift constitute two independent signals mixed in linear manner. Daub-6 mother wavelet is used for carrying out the dyadic wavelet packet decomposition of the acquired signal [6].The algorithm computes wavelet packet coefficients and then in each scale the energy of the signal is calculated.The energies of the signal for both the detailed \& coarse levels are calculated in each scale using the wavelet coefficients [7]. These energies represent the energy of the decomposed signal in assumed scales as

$$
E_{a, b}^{d}=\sum_{b=-\infty}^{\infty}\left|d_{a, b}\right|^{2}, E_{a, b}^{c}=\sum_{n=-\infty}^{\infty}\left|c_{n}\right|^{2}
$$

In these equations (1), $E_{a, b}^{d}$ represents the energy in the detail level of the signal at scale a (high-pass filtering branch) and $E_{a, b}^{c}$ represents the energy of the signal in the coarse level of scale a (low-pass filtering branch). After comparing these energy levels, the branch of the binary tree that has the higher energy is chosen. The path for higher energy branches is to be followed until the present energy differences do not exceed threshold level of $\mathrm{E}_{\mathrm{th}}$. As the binary tree search is completed, in order to retrieve baseline wander signal, inverse wavelet transform of the wavelet packet coefficients of the last scale is taken [7]. In order to suppress the baseline drift, estimated baseline wander is subtracted from original PPG signal. Thus, a baseline wander free PPG signal is identified. For threshold level selection, normalized energy of the signal in every scale is calculated and checked if value of energy is $0.1 \%$ of the total energy of the signal or not, till reaching the last scale [8].

\section{Analysis}

The Analysis section has been divided into two parts, first being time domain analysis and second being Frequency domain analysis. Time domain analysis has been done by two different methods, Parameter Extraction and Poincare Plotting, whereas Frequency domain analysis has been done using Power Spectral Density (PSD) method.This analysis will help in computation of various indices for classification of diseases.

\subsection{Parameter Extraction}

Extraction of the characteristic points or features from the PPG signal is done for estimation of various parameters..

Heart Rate (HR) The aindex is used to calculate the heart rate of subject.

$$
\text { Heart Rate }=\frac{1}{(A-A \text { interval in sec. })} * 60
$$

Pulse Transit Time (PTT) PTT is defined as the time interval between two characteristic points the R peak of the electrocardiogram (ECG) and the peak of the pulse at finger. In this paper, it is defined as, the time interval between the peak of ECG and Peak of finger PPG respectively [9].

Pulse Wave Velocity (PWV) Pulse Wave Velocity (PWV) is related to BP, the cubage and flexibility of the arteries. Thus PWV, a potential tool, is useful and a convenient parameter in continuous monitoring of Blood Pressure [9].

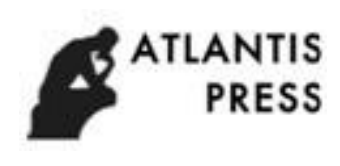




$$
P W V(\mathrm{~cm} / \mathrm{ms})=\frac{B D C * H \operatorname{eight}(\mathrm{cm})}{P T T(m s)}
$$

$\mathrm{BDC}=$ body correlation factor, and height $=$ body length. $\mathrm{BDC}$ is 0.5 for adults when taken the finger for detection of the peripheral pulse wave as used.

Stiffness Index (SI) Stiffness index (SI) is a measure of larger artery stiffness, can be used as a surrogate measure of PWV, correlates negatively with age. SI is calculated in terms of the subject's height divided by pulse transit time (PTT or $\Delta \mathrm{T})$.

where $\mathrm{H}$ is the subject's height [4].

$$
S I=\frac{H}{\Delta T}
$$

\subsection{Poincare Plot}

Poincare plot (PP) is a visual technique based on quantitative method for applying on short or long PPG signals, and it uses the capability of human eye to visualize patterns by introducing various parameters (descriptors) for quantifying information from such plot [10]. Thus a reasonable Poincare plot is extraction of information from a long or a short PPG signal that may contain many outliers or artifacts. PP is therefore superior technique to Discrete Fourier Transform method that requires treating of outliers and artifacts for reliable results [10]. Two auxiliary vectors for a data vector given below $A A=\left(\right.$ AAindex $_{1}$, AAindex $_{2}$, AAindex $_{3}, \ldots \ldots . . . . . .$, AAindex $\left._{N}\right)$ can be defined as:

$$
\begin{aligned}
& A A^{+}=\left(\text {AAindex }_{1}, \text { AAindex }_{2}, \ldots . ., \text { AAindex }_{N-1}\right) \\
& A A^{-}=\left(\text {AAindex }_{2}, A \text { Aindex }_{3}, \ldots . . . ., \text { AAindex }_{N}\right)
\end{aligned}
$$

These are known as AAin \& AAin+1 in medical literature. The PP has all ordered pairs: $\left(A A_{i}^{+}, A A_{i}^{-}\right)$,

$$
\mathrm{i}=1, \ldots \ldots \ldots \ldots, \mathrm{N}-1
$$

When this procedure is used on the PPG signal of a healthy person, a comet like shapeis derived. The Fig 2 shows Poincare plot output descriptors from PPG signal collected from a healthy person.SD2 is defined as standard deviation of projection of PP on the line of identity $(\mathrm{y}=\mathrm{x})$ and SD1 is standard deviation of projection of PP on the line perpendicular to the line of identity $(\mathrm{y}=-\mathrm{x})$ [10].It is defined as:

$$
S D 1=\sqrt{\operatorname{Var}\left(x_{1}\right)}, \quad S D 2=\sqrt{\operatorname{Vak}\left(x_{2}\right)}
$$

Where $\operatorname{Var}(\mathrm{x})$ is variance of $\mathrm{x}$ and

$$
x_{1}=\frac{A A^{+}-A A^{-}}{\sqrt{2}}, \quad x_{2}=\frac{A A^{+}+A A^{-}}{\sqrt{2}}
$$

SD1 represents short term variability \& SD2 represents both short term \& long term variability. Total variability as measured by PP can be given by area of ellipse with axes (SD1, SD2) centered on $\left(\overline{A A}^{+}, \overline{A A}^{-}\right)$:

$$
\text { area }=\pi \times S D 1 \times S D 2
$$

\subsection{Power Spectral Density}

Power spectral density function (PSD) shows the strength of the variations (energy) as a function of frequency.The unit of PSD is energy per frequency and one can obtain energy within a specific frequency range by integrating PSD within that frequency range. Computation of PSD is done directly by the method called FFT and then transforming it.

The PPG signal acquired from acquisition system over a period of 180 seconds consists of short term Variability in Heart Rate (HRV) \& Peripheral Blood Flow (PBFV). Multiple rhythms are affecting these variations making the time domain data is difficult to analyze. Power Spectral Density (PSD) calculated from Fast Fourier Transform (FFT) isolates such rhythms \& presents the time domain data distinctly [11]. 


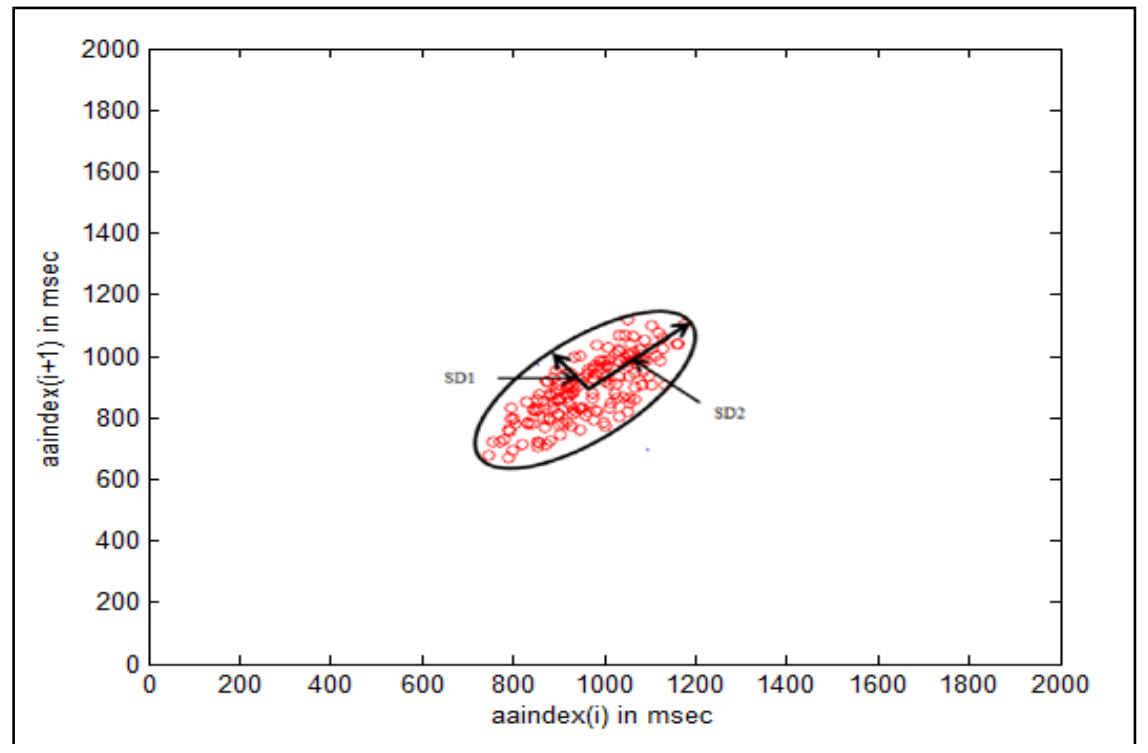

Fig.2. Poincare plot

The power spectral density has been assesed in three frequency band for biomedical signals viz, VLF ( $0-0.08 \mathrm{~Hz})$, LF $(0.04-0.16 \mathrm{~Hz})$ and HF $(0.16-0.4 \mathrm{~Hz})[11]$. From FFT three distinct peaks centered at $0.04,0.22,0.44 \mathrm{~Hz}$ are seen as Very Low Frequency (VLF) peak, Low Frequency (LF) peak, High Frequency (HF) peak respectively. The graphs in each case are clearly distinguished. But the similar measurements when carried out on more subjects, a clear logical mapping of graph \& type of ailment the subjects are facing, was not achievable. Therefore four areas were drawn from each PSD output using 3 distinct peaks centered at $0.04,0.22$, and $0.4 \mathrm{~Hz}$ respectively as shown in Fig. 3.

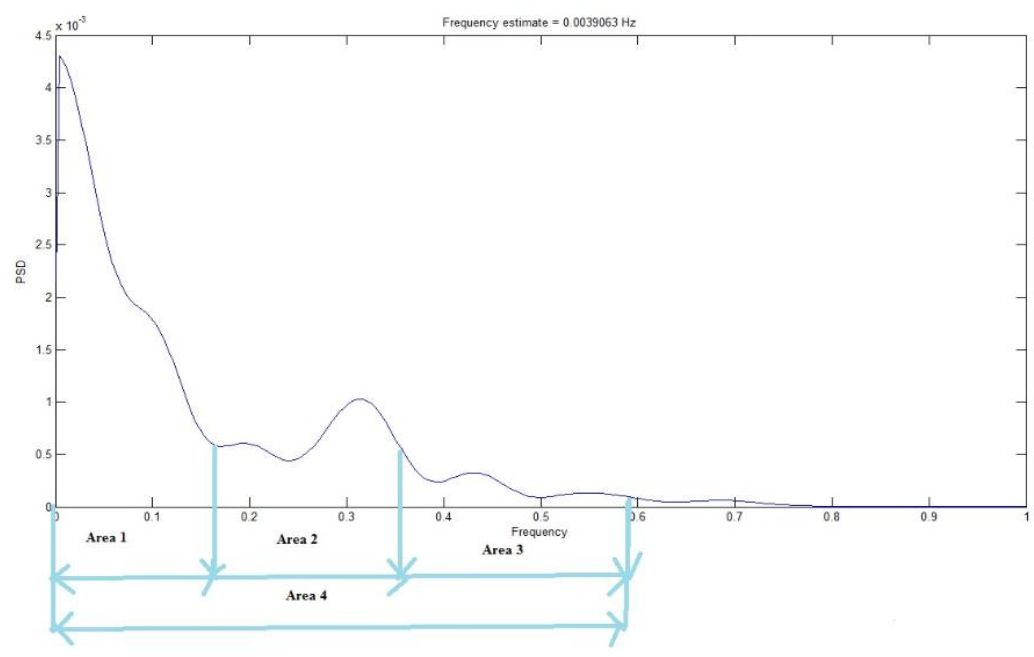

Fig.3. Power Spectral Density

The AA index is used to compute the area of PSD as follows:

$>$ Area1=area under the curve of PSD from $0-0.16 \mathrm{~Hz}$.

$>$ Area2=area under the curve of PSD from $0.16-0.35 \mathrm{~Hz}$.

$>$ Area3=area under the curve of PSD from 0.36.-0.6Hz.

$>$ Area4=area under the curve of PSD from 0-0.69 Hz. 


\subsection{Classification}

Parameter Extraction, PSD, Poincare Plot, it is not reasonably possible to classify the subjects using any of these individual methods of analysis. Therefore multiple combinations of outputs from PSD \& Poincare Plot were tried by using multi regression analysis by forming equations to reach 3 logical indices that could represent different ailments.Equation 9,10,11 indicates Index 1, Index 2, and Index 3 respectively.

Index $1=$

$$
(1.849) * H R+(0.972) * S D 1+(2.282) * S D 2+(4.491) S D R R+\quad(-0.040) *
$$

Area of Ellipse $+(15.546) *$ mean $+(-14.287) *$ mean $\quad(-0.849) *$ Centroid $+(17.559) *$ Area $1+$ $(0.706) *$ Area $2+\quad(-2.020) *$ Area $3+(19.083) *$ Area 4.

Index $2=$

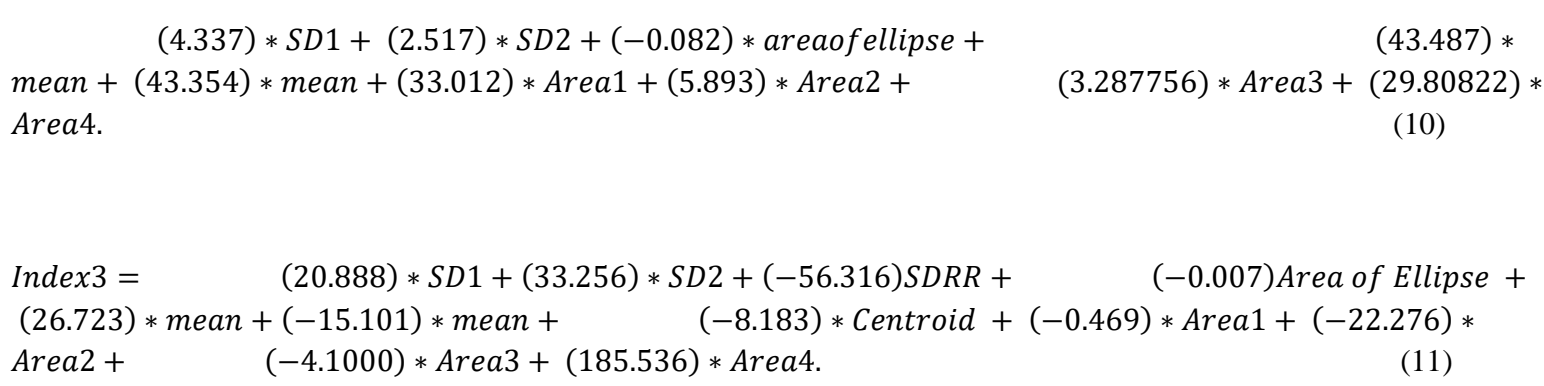

\section{Results and Discussions}

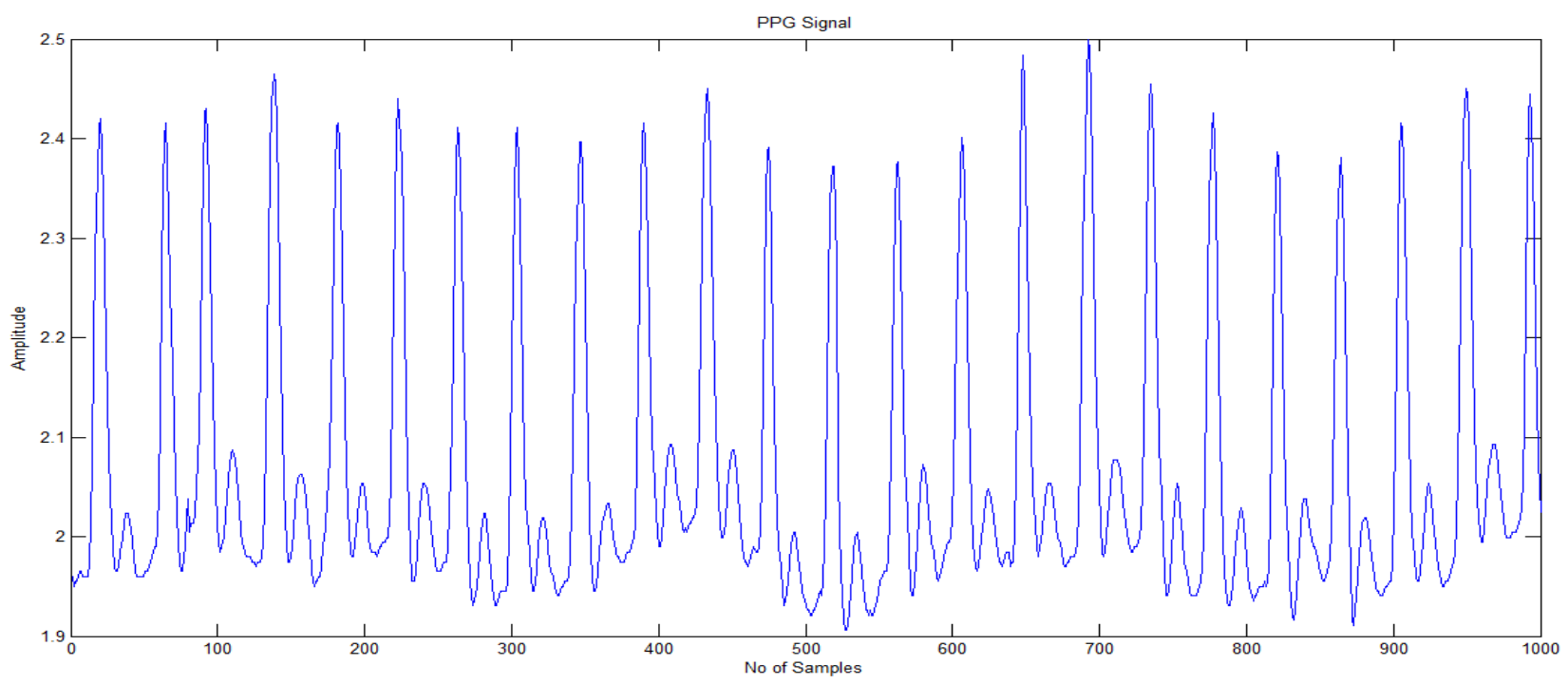

Fig 4. Original PPG signal acquired in MATLAB 


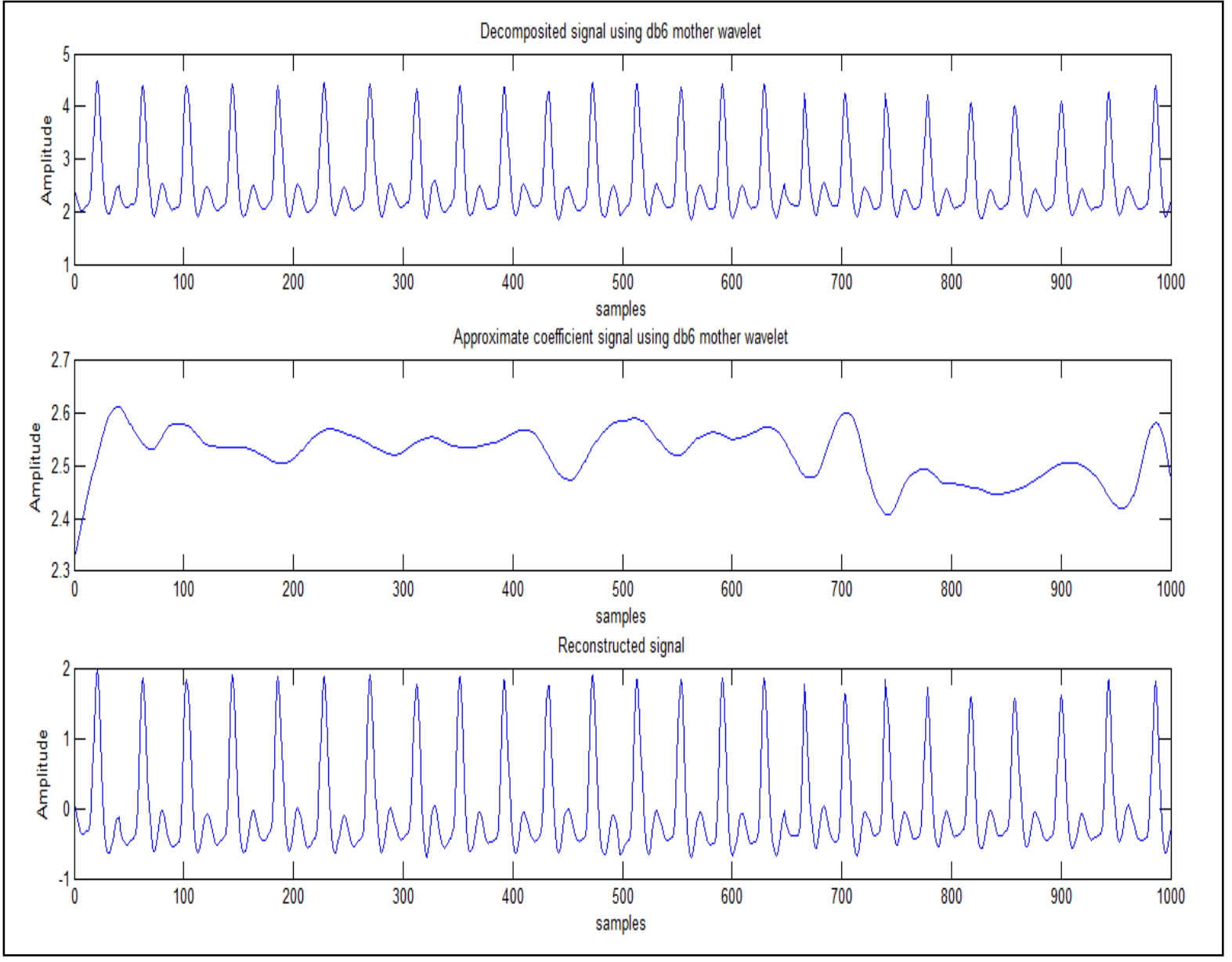

Fig.5. Decomposition and Reconstruction of PPG signal by applying wavelet transform using 'db6' mother wavelet

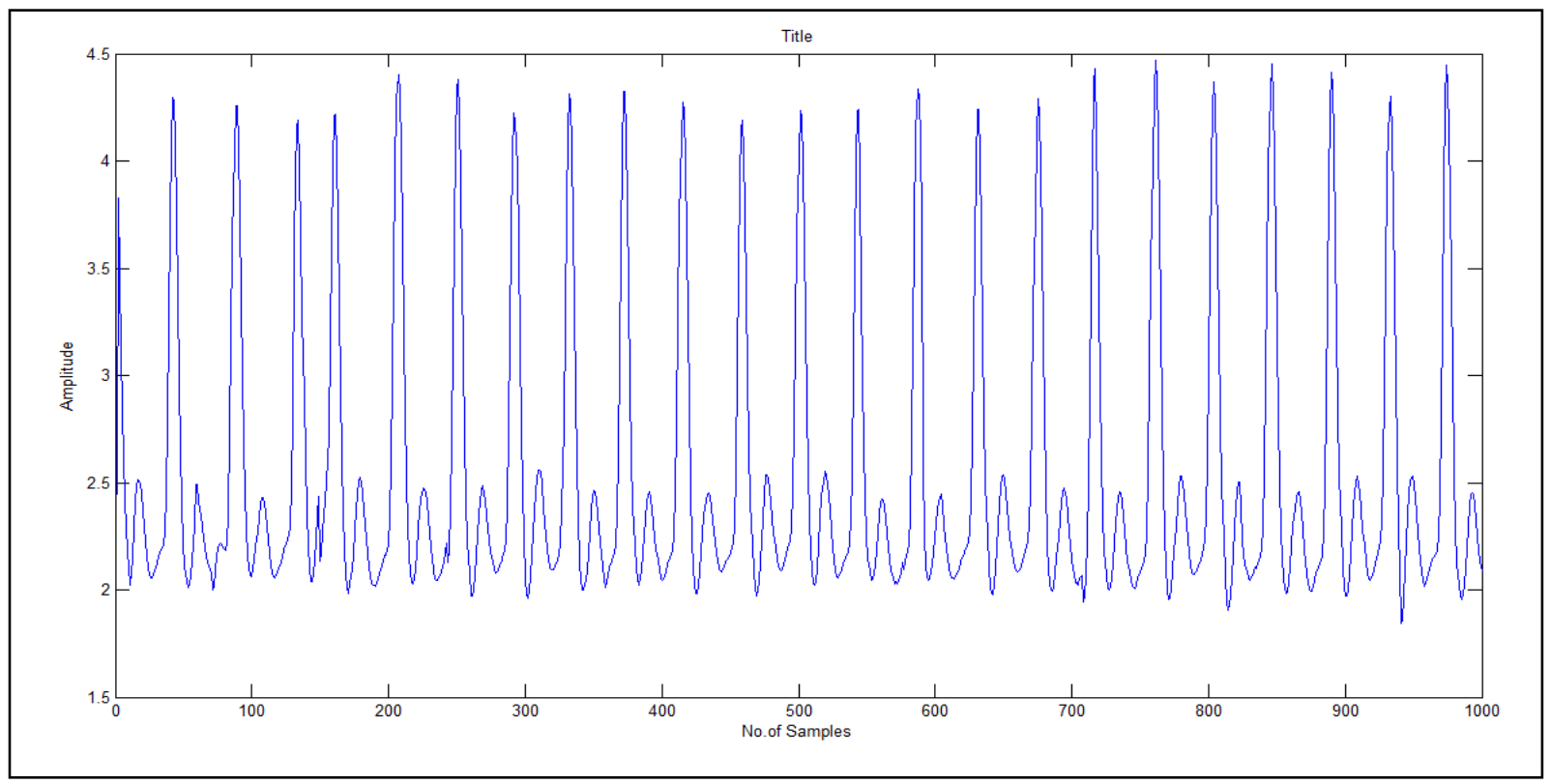

Fig.6. Smoothened and Processed PPG signal 


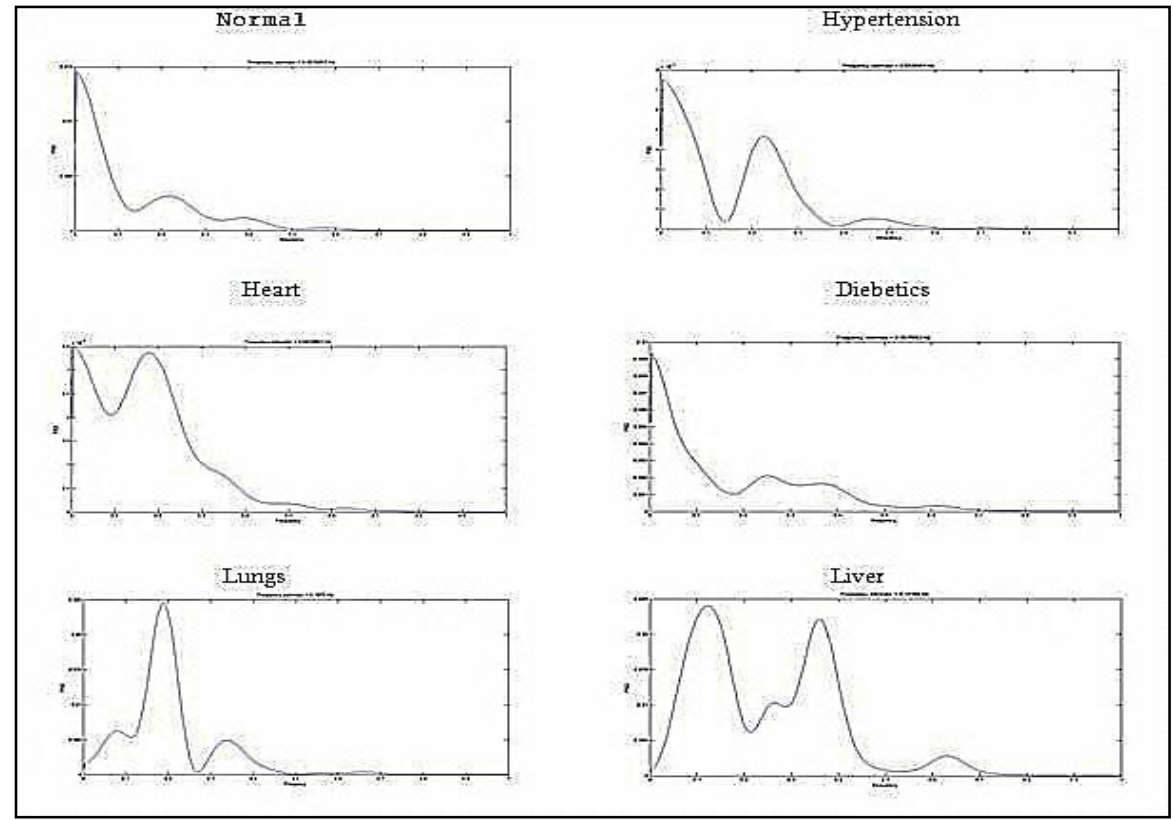

Fig.7. Power Spectral Densities

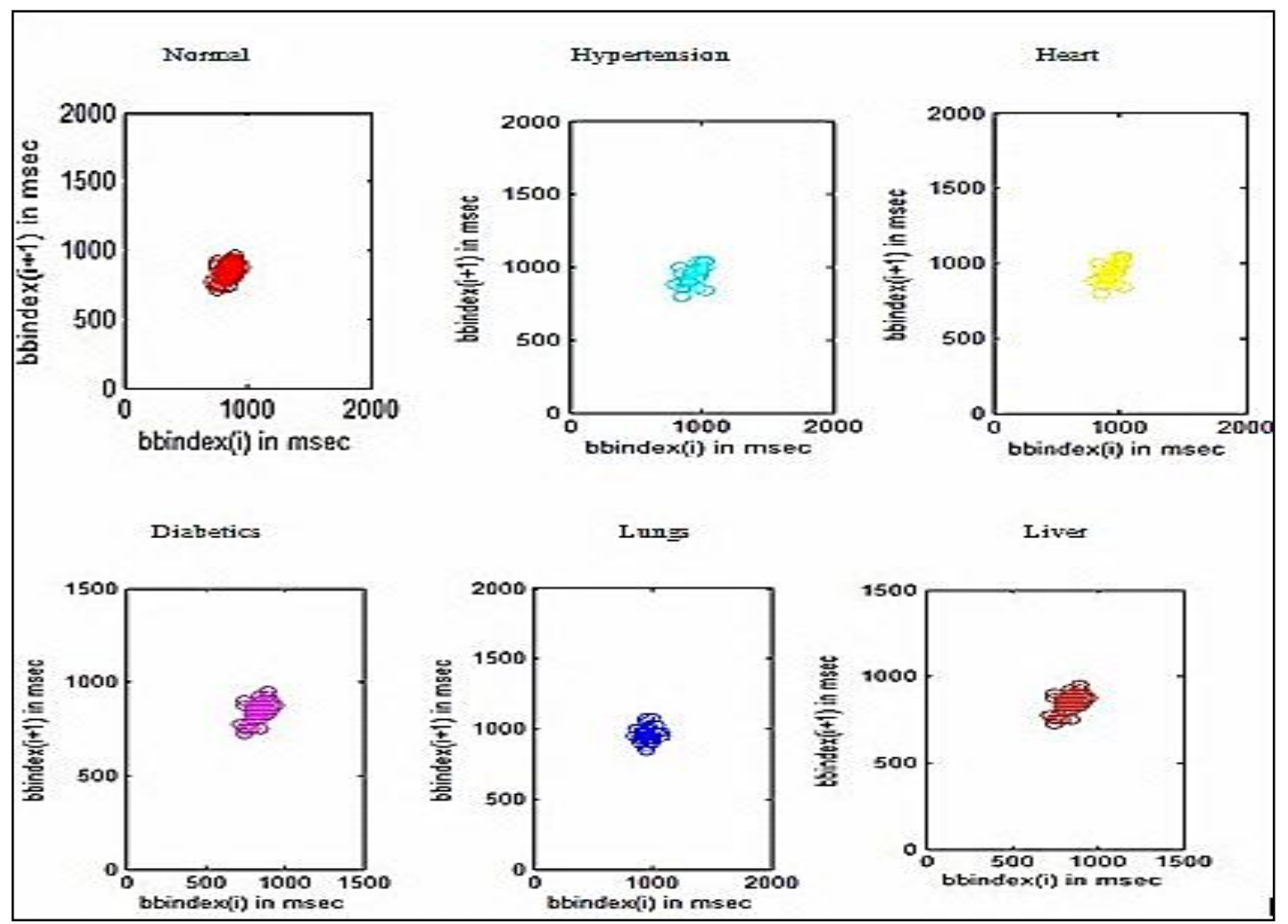

Fig.8. Poincare plots of subjects with ailments and without ailments

The Fig. 4 and 6 shows the acquired and processed and smoothened PPG signal Similarly ECG signal is also acquired and processed in MATLAB.Fig 5 shows Decomposition and Reconstruction of PPG signal by applying wavelet transform using ' $\mathrm{db} 6$ ' mother wavelet. Table 1 shows the parameters calculated for various subjects with different ailments.Fig 7 and 8 shows Power Spectral Densities and Poincare plots of subjects with ailments and without ailments. 
Table 1. The parameters calculated for various subjects with different ailments

\begin{tabular}{|c|c|c|c|c|c|}
\hline \multirow{2}{*}{ Type of ailment } & Subject & $\begin{array}{c}\text { HR } \\
\text { beats/min }\end{array}$ & $\begin{array}{c}\text { PTT } \\
(\mathbf{~ m s}) \\
(\mathbf{W}-\mathbf{F})\end{array}$ & $\begin{array}{c}\text { PWV } \\
(\mathbf{m} / \mathbf{s e c}) \\
\mathbf{( W - F )}\end{array}$ & $\begin{array}{c}\text { Stiffness Index } \\
(\mathbf{c m} / \mathbf{s e c})\end{array}$ \\
\hline \multirow{2}{*}{ Normal } & Subject 1 & 71 & 88 & 1.02 & 3.011 \\
\cline { 2 - 6 } & Subject 2 & 91 & 96 & 1.88 & 3.081 \\
\hline \multirow{2}{*}{ Cardiac } & Subject 1 & 84 & 54 & 2.57 & 5.124 \\
\cline { 2 - 6 } & Subject 2 & 67 & 52 & 2.26 & 5.28 \\
\hline \multirow{2}{*}{ Diabetic } & Subject 1 & 76 & 67 & 1.93 & 6.38 \\
\cline { 2 - 6 } & Subject 2 & 70 & 66 & 1.78 & 4.41 \\
\hline \multirow{2}{*}{ Lungs } & Subject 1 & 71 & 103 & 1.84 & 7.35 \\
\cline { 2 - 6 } & Subject 2 & 81 & 110 & 1.64 & 5.508 \\
\hline \multirow{2}{*}{ Liver } & Subject 1 & 85 & 94 & 2.23 & 4.123 \\
\cline { 2 - 6 } & Subject 2 & 99 & 98 & 1.93 & 3.770 \\
\hline
\end{tabular}

Classification The logical indices serve as a preliminary level diagnosis tool for classification of various diseases. Table 2 shows the classification of diseases like Normal, Cardiac, Diabetic, Liver, and Lungs. The three indices determination is based on the no. of parameters and no. of subjects with and without different ailments. So in order to get the satisfactory results more no. of patients and no. of parameters should be considered.

Table 2 Classification of diseases

\begin{tabular}{|c|c|c|c|}
\hline Type of ailment & Index 1 & Index 2 & Index3 \\
\hline Normal & $<100$ & NA & NA \\
\hline Cardiac & $>100$ & $<200$ & $<20$ \\
\hline Diabetic & $>100$ & $<200$ & $>20$ \\
\hline Liver & $>100$ & $200-450$ & NA \\
\hline Lungs & $>100$ & $>450$ & NA \\
\hline
\end{tabular}

First Index is used to classify normal and abnormal subject. The criteria to distinguish between subjects was observed to be value of Index 1 which if less than 100 then indicates subject as normal else abnormal. Abnormal subjects can then be categorized in two groups based on Index 2 such as risk of hypertension/heart \& Diabetics and Lungs and Liver Diseases. If value of Index 2 less than 200, it indicates risk of Hypertension/Heart and Diabetic diseases and If value of Index 2 greater than 200, it indicates risk of Lungs and Liver diseases. If value of Index 2 is in the range of 200-450 then there is risk of Liver diseases \& value greater than 450 indicates risk of Lungs related risks. Index 3 is developed to discriminate the subjects facing Hypertension/Heart or Diabetic diseases. If value of index 3 is less than 20 then it indicates a risk of Hypertension/Heart diseases and greater than 20 indicates a risk of Diabetic disease.

\section{Conclusion}

In Biomedical measurement, there are very few reliable and suitable non-invasive methods (ECG) for measuring and monitoring cardiac flow in clinical practice. Photo plethysmography method is able to further enhance this reliability. This paper discusses about various hemodynamic variables which can be extracted from the PPG signals. A noninvasive method to measure and analyze these hemodynamic variables would assist doctor with essential diagnostic tools for patients care with critical hemodynamic disorders. The proposed prototype PPG system can be used to capture the pulsations of the flow of blood in the arteries along with ECG signal. However, further improvements in motion noise reduction as well as analysis capabilities are required. For 
demonstration of its functionality, PPG signals from 33 individuals are taken. Parameter extraction and HRV signal analysis of the acquired PPG signals was carried out using MATLAB and a preliminary level of diagnosis of diseases is proposed using frequency-domain and time-domain characteristics. This classification uses multiple regression analysis to discriminate between subjects with and without ailments. With few patients one cannot come up with satisfactory results. Hence, better refining of classification should be done with more number of subjects.

\section{References}

[1] S. A. Mandlik, G.D. Jindal, T.S. Ananthakrishnan, S.K. Kataria, V. Sinha, R. K. Jain, S.K. Singh, A.R. Kini, A. K. Deshpande, "Anu-Photo Rheography for Applications in Clinical Medicine", research thesis, 2004

[2] E. Gil, M. Orini, R. Bailon, J. M. Vergara, L. Mainardi and P. Lagunal,” Photoplethysmography pulse rate variability as a surrogate measurement of heart rate variability during non-stationary conditions" , Physiol. Meas., vol. 31,pp 1271-1290,2010.

[3] J. Allen,"Photoplethysmography and its application in clinical physiological measurement", Physiol. Meas., vol. 28 pp. R1-R39, 2007.

[4] M. Elgendi," On the Analysis of Fingertip Photoplethysmogram Signals", Current Cardiology Reviews, vol. 8, pp.14-25, 2012.

[5] Pranali C. Choudhari, Dr. M. S. Panse, (2014), “Artifact Removal from the Radial Bioimpedance Signal using Adaptive Wavelet Packet Transform”, International Journal of Computational Engineering Research, Vol 4 (7), pp 95- 101, July 2014.

[6] Choudhari, Pranali C., and M. S. Panse, "Denoising of Radial Bioimpedance Signals using Adaptive Wavelet Packet Transform and Kalman Filter", IOSR Journal of VLSI and Signal Processing (IOSR-JVSP) Volume 5, Issue 1, Ver. II (Jan - Feb. 2015), PP 01-08 e-ISSN: 2319 - 4200, p-ISSN No. : 2319 - 4197.

[7] Pranali C. Choudhari, M. S. Panse, "Intelligent System Based on Impedance Cardiography for Non-invasive Measurement and Diagnosis", Advances in Intelligent Systems and Computing, Volume 384, (2016), pp 69-78.

[8] Tinati, M. Ali, and B. Mozaffary, "A wavelet packets approach to electrocardiograph baseline drifts cancellation.” International journal of biomedical imaging Vol. 2006, Article ID 97157, Pages 1-9.

[9] Teja, B.R., "Calculation of Blood Pulse Transit Time from PPG." B.tech Thesis, National Institute of Technology Rourkela, 2012.

[10] J. Piskorski, P. Guzik," Filtering Poincaré plots", computational methods in science and technology ,vol. 11, pp. 39-48,2005.

[11] Reyes, H. Nazeran, M. Franco, E. Haltiwanger," Wireless Photoplethysmographic Device for Heart Rate Variability Signal Acquisition and Analysis" presented at 34th Annual Int. Conference of the IEEE EMBS San Diego, California USA, 2012. 